\section{Use of Methanol containing Boron Trifluoride for the Esterification of Unsaturated Fatty Acids}

ATtentron was recently directed ${ }^{x}$ to the production of considerable amounts of methoxy-substituted fatty acids as artefacts during the esterification of unsaturated fatty acids with methanol containing about 50 per cent of boron trifluoride.

However, when the boron fluoride content is reduced by dilution with methanol, progressively smaller proportions of methoxy-substituted derivatives of unsaturated fatty acid methyl esters are formed; when esterification is carried out for $2 \mathrm{~min}$ under reflux using a solution containing 12.5 per cent boron fluoride as advocated by Metcalfe and Schmitz $^{2}$, no methoxy-substituted derivatives are produced. Though methanol containing boron fluoride is satisfactory for the esterification of unsaturated fatty acids under carefully controlled conditions, tho possibility that methoxy-substituted products may arise inadvertently should, nevertheless, be recognized.

\section{A. K. Lovgr}

Rowett Research Institute,

Bucksburn, Aberdeen.

${ }^{1}$ Lough, A. K., Biochem. J., 90, 4, C (1964).

${ }^{2}$ Metcalfe, L. D., and Schmitz, A. A., Anal. Chem., 33, 363 (1961).

\section{Continuous Flow Spotting for Paper Chromatograms}

IN spite of the claims of other systems, paper chromatography remains a major analytical tool of forensic toxicology. One of its minor disadvantages is that, in order to get sufficient material on to the paper to give an identifiable spot, a dilute solution must be applied by the tedious process of 'spotting' small aliquots and allowing them to dry between each application. We wish to report a very simple and reliable method in which the solution is allowed to flow continuously on to the paper, producing a spot about $0.5 \mathrm{~cm}$ in diameter.

The device (Fig. $1 A$ ) is made by pulling out a piece of glass tubing ( $6 \mathrm{~mm}$ internal diameter) into a capillary, which is bent into $U$-form as shown. A convenient length for the wider limb is $3 \mathrm{~cm}$, the capillary being about $0.5 \mathrm{~cm}$ longer. The latter has a diameter of about $1.5 \mathrm{~mm}$, and narrows at the tip to an orifice of $0.2-0.3 \mathrm{~mm}$. Too fine an orifice delivers the liquid too slowly and may make holes in the paper; too coarse a capillary will not maintain the flow of liquid.

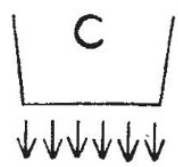

B

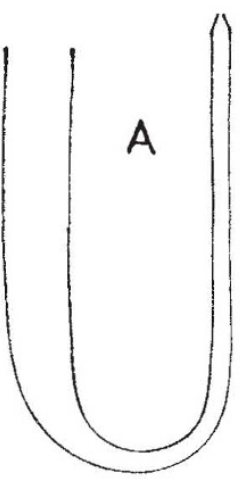

Fig. 1
The device is filled with the solution to be spotted, and the capillary 'primed' if necessary by tipping it or by applying pressure to the end of the wider limb. It is placed with the orifice $2-3 \mathrm{~mm}$ below a sheet of chromatography paper, $B$, suspended horizontally between supports. A hot air blower, $C$, is arranged immediately above the paper as shown. When the blower is switched on, the paper is depressed by the air stream, and touches the top of the capillary. The solution is drawn into the paper by capillary attraction, and rapidly evaporates in the blast of hot air, thus limiting the size of the spot produced. Once started, the apparatus may be left unattended, as, should the blower stop, the paper springs away from the capillary, thus obviating any risk of flooding.

With ethanol or chloroform, a spot 3-5 $\mathrm{mm}$ in diameter is obtained; with water the spot is considerably larger, being about $1 \mathrm{~cm}$ or more in diameter. The volume spotted on the paper in a given time depends on the exact dimensions of the device, but, in general, $0.2 \mathrm{ml}$. of an ethanolic solution is run on in $5 \mathrm{~min}$. Using the citrate-butanol system of Curry and Powell ${ }^{1}$, a I-min run with the device filled with a 0.01 per cent solution of morphine, nicotine. strychnine, cocaine or chlorpromazine produces spots easily visible when sprayed with iodoplatinate solution.

E. G. C. Clarke

SHeIlA A. Sowter

Department of Physiology,

Royal Veterinary College, London, N.W.1.

${ }^{1}$ Curry, A. S., and Powell, M., Nature, 173, 1143 (1954).

\section{Gas-phase Ion-molecule Interactions involving Atom Transfer; Limitations of the Orbiting Theory in accounting for the Variation of Cross-section with Energy}

ION-MOLECULE interactions of the type $\mathrm{X}^{+}+Y \mathrm{H}=$ $\mathrm{XH}^{+}+Y$ are of interest in gas kinetics since they are chemical reactions in which the translational energy of one of the reactants may be chosen over a wide range. This may be achieved by accelerating an ion beam to the desired velocity. However, most of the information on such reactions has in fact been derived by supplying a mixture of the gases $X$ and $Y H$ to the source of a mass spectrometer, and, if possible, choosing the electron energy so that $X$ (but not $Y H$ ) is ionized ${ }^{1}$. The ultimate translational energy of $X^{+}$is then determined by its initial position and the potential difference between the repeller plate and the exit slit of the source. This technique involves the acceleration of ions throughout the actual resction zone, and the observed reaction oross-sections correspond to mean values for all the ions with translational energies up to the maximum. A rigorous interpretation of the effect of translational energy on the reaction cross-section is, therefore, difficult if the 'mixed gas' approach is used.

The mechanism of such reactions is believed to involve the initial formation of an orbiting complex between $X^{+}$ and $Y H$, followed by transfer of the hydrogen atom. An orbiting complex is formed if the attractive polarization force between the ion and the molecule is equal to, or greater than, the centrifugal force which tends to dissociate the complex. The maximum impact parameter (the impact parameter is the perpendicular distance botween one particle and the initial line of relative motion of the other) which can lead to the formation of an orbiting complex is taken as the radius of the reaction crosssection $^{2}$. Thus the reaction cross-section $(\sigma \nabla)$ for an interaction between an ion and a molecule with an initial relative velocity $V$ is deduced to be $(\pi / V)\left(4 e^{2} \alpha / \mu\right)^{\frac{1}{3}}$, where $e$ is the charge of the ion, $\alpha$ th, polarizability of the molecule, and $\mu$ the reduced mass of the system. 